\title{
Feasibility and acceptability of financial incentives and peer-led life skills training for improving adherence to antiretroviral therapy among adolescents living with HIV in Rwanda: A pilot study
}

\section{Majdi Osman}

Youth Development Labs (YLabs)

\section{Simon Pierre Niyonsenga}

Rwanda Biomedical Centre

\section{Manasi Sharma}

Harvard University T H Chan School of Public Health

\section{Rebecca Hope}

Youth Development Labs (YLabs)

Shannon Milroy

Youth Development Labs (YLabs)

Jordan Levine ( $\square$ jordan.levine@ylabsglobal.org )

Youth Development Labs (YLabs) https://orcid.org/0000-0002-3008-9507

\section{Caroline Kusi}

Youth Development Labs (YLabs)

\section{Tiffany Chan}

Harvard University T H Chan School of Public Health

\section{Patient Ngamije}

Kirehe District Hospital

\section{Bernard Kabayiza}

Centre Hospitalier Universitaire de Kigali

\section{Olivier Manzi}

Centre Hospitalier Universitaire de Kigali

Muhayimpundu Ribakare

Rwanda Biomedical Centre

\section{Laurence Isaaya Ntawunga}

University of Rwanda

Jean-Luc Nkurikyamfuma

Centre Hospitalier Universitaire de Kigali

\section{Lameck Habumugisha}

Youth Development Labs (YLabs)

\section{Eric Remera}

Rwanda Biomedical Centre

\section{Sabin Nsanzimana}

Rwanda Biomedical Centre 


\section{Research article}

Keywords: Rwanda, adolescents, HIV/AIDS, Viral suppression, Medication adherence, Financial incentives, Life skills Posted Date: April 24th, 2020

DOI: https://doi.org/10.21203/rs.3.rs-23345/v1

License: (c) (i) This work is licensed under a Creative Commons Attribution 4.0 International License. Read Full License 


\section{Abstract \\ Background}

The Human Immunodeficiency Virus (HIV) is the leading cause of death among adolescents in sub-Saharan Africa. Despite the long-term benefits of antiretroviral therapy (ART), adherence remains low among adolescents due to challenges related to cost, acquisition, and treatment regimen. Sub-optimal adherence to ART is associated with the development of viral resistance, treatment failure, and increased morbidity and mortality. Financial incentives and life skills training interventions have shown early promise in motivating protective behaviors, however, gaps still remain around effective and innovative ways to motivate adolescents to stay in HIV care. In partnership with youth, we developed an intervention to address their social and financial pressures in order to promote adherence.

\section{Methods}

A human-centered design approach was utilized to engage adolescents in the initial design of the intervention. Through random sampling, 72 adolescents ages 12-19 were then recruited from two clinics in Rwanda for the pilot study. Adolescents participated in three monthly peer-led life skills trainings at clinics and received financial incentives via mobile money upon clinic attendance and demonstration of suppressed viral load. Semi-structured interviews were then conducted with adolescents, healthcare workers, and caregivers to understand their experiences with the intervention.

\section{Results}

Participants responded favorably to the intervention because of the psychosocial, financial, and health benefits it provided. Caregivers felt that adolescents' moods, attitudes, and overall well-being improved over time. Adolescents used funds to purchase school supplies and save for investments, thus mitigating their financial burdens. Additionally, we learned that logistical challenges such as SIM card registration and mobile phone access must be considered for scaleup of the intervention.

\section{Conclusions}

To our knowledge, this is the first intervention designed in partnership with youth in sub-Saharan Africa aimed at removing financial barriers to ART adherence. The findings suggest that involving youth in the design of programs, providing them with financial incentives, and training them to use and invest their money wisely has a positive effect on them and their families. Thus, to improve adherence to ART and retention in care among HIV-positive youth, it is critical to address their socioeconomic and psychosocial needs.

\section{Background}

Globally, 1.6 million adolescents ages 10-19 are living with the Human Immunodeficiency Virus (HIV). Eighty-nine percent of this population resides in sub-Saharan Africa [1]. Despite the introduction of antiretroviral therapy (ART), HIV is still the leading cause of death among adolescents in this region [1]. ART adherence is critical for sustained viral suppression to maintain immune function, which in turn reduces risk of infection and mortality [2]. Conversely, suboptimal adherence to ART is associated with immunosuppression, development of viral resistance, treatment failure, and increased morbidity and mortality [3]. Several studies conducted in resource-limited settings have found that adherence to ART is lower in adolescents than in the adult population [4-6]. Adolescence is a crucial time for cognitive maturity, identity formation, establishment of independent social relationships, and transition to an autonomous lifestyle [7, 8]. 
This developmental phase is often inconsistent with the level of responsibility and discipline necessary to meet the demands of a daily treatment regimen.

Adolescence is characterized by the development of cognitive maturity, identity formation, establishment of independent social relationships, and transition to an autonomous lifestyle [7, 9]. Neurodevelopmental research suggests that areas of the brain stimulated by rewards, namely the ventral striatum, medial orbitofrontal cortex, and medial prefrontal cortex [10, 11] reach peak activation in adolescence. As such, adolescents have a heightened response to immediate, small rewards over larger, long-term gains [12]. HIV medication regimens involve taking several tablets every day. For many adolescents, skipping tablet-taking on a given day, when they feel well and can be temporarily unencumbered by their diagnosis, is a more appealing immediate reward than the prospect of remaining healthy in the future. Furthermore, as adolescents develop their identity, those living with HIV may struggle to incorporate their illness and the permanent changes to their lifestyle that it mandates [7].

Despite having a theoretical understanding of the consequences of non-adherence, adolescents may see such behavior as a way of testing limits and experimenting [7]. Overprotective parenting may conversely encourage adolescents to rebel by not taking their medication, missing medical appointments, or ignoring dietary restrictions suggested in their treatment regimen [7]. This allows adolescents more perceived control over their personal schedules, relief from medication side effects, an increase in savings from not purchasing medication, and temporary freedom from thinking about their illness [7].

Poverty creates additional challenges for both curbing HIV transmission and promoting treatment adherence.

Adolescents facing extreme financial instability are especially vulnerable to risky behaviors that increase access to quick cash, such as transactional sex, which can further promote the spread of HIV [13]. Scarce employment opportunities, low household wealth, and, in HIV-prevalent environments, unstable guardianship following parental illness or death from HIV/AIDS, limit adolescents' ability to meet their basic needs and save money for medication [14-18].

Financial incentives have been previously utilized to promote both HIV prevention behaviors and ART adherence [19, 20]. Researchers in South Africa demonstrated that access to cash through social protection schemes mitigated risk-taking behavior among adolescents [21]. In Nigeria, cash transfers that incentivized adolescent girls to remain in school reduced risky behavior and HIV acquisition [22]. Furthermore, a randomized controlled trial in Uganda showed that cash transfers of \$5-8 per month to cover transportation costs to an HIV clinic increased treatment adherence among adult patients [23]. These studies led us to test the hypothesis that financial incentives may be similarly successful in promoting treatment adherence among adolescent patients.

Peer influence has also been found to be an important factor in adopting behaviors that support ART adherence among adolescents [18]. Peer-led HIV programs and trainings have a positive effect on knowledge, attitudes, normative beliefs, and self-efficacy, all of which are critical in facilitating behavior change [24, 25]. Peer education has been previously leveraged to increase HIV prevention tactics, including testing, counseling, and condom usage among adolescents in South Africa and Ethiopia [26, 27]. The prioritization of peer-led trainings in interventions can offer adolescents knowledge on various life topics and promote positive behaviors more generally.

Evidence for the effectiveness, feasibility, and acceptability of combined financial incentives and peer-led life skills training among adolescents is limited. Adolescents are often not involved in cash transfer programs because they are perceived to have poor judgment or be more likely to experience coercion. In partnership with youth, we developed an integrated financial incentives and peer-led life skills training program called YBank to improve ART adherence among adolescents living with HIV in Rwanda. Our pilot study aimed to assess the feasibility and acceptability of the YBank program among adolescents, caregivers, and clinicians. 
We hypothesized that financial incentives in the form of cash transfers, partnered with a peer education and support program, may help to address key barriers faced by adolescents living with HIV in three distinct ways. First, cash transfers help to cushion the social and economic impact of HIV by addressing short-term financial barriers to service access and adherence to treatment regimens. Second, peer-led life skills training helps adolescents to cultivate relationships among peers, use social skills prudently, and foster long-term resilience as they build helpful life proficiencies. Finally, the use of rewards can mitigate behavioral biases, such as present bias (preferring short- to longterm rewards) and default bias (preferring current to new behaviors), which may hinder adherence.

\section{Human-centered design methodology}

A human-centered design approach was employed to engage adolescents living with HIV and key stakeholders in the design of the program. We conducted focus group discussions and semi-structured interviews with 16 adolescents ages 12-19 living with HIV who were enrolled in care. We also interviewed 14 caregivers of adolescents with HIV and five healthcare providers working at Centre Hospitalier Universitaire de Kigali (CHUK), an HIV clinic in urban Kigali, Rwanda. Through these sessions, we sought to understand barriers to adherence, perceptions of clinical services, acceptability and feasibility of peer-led life skills trainings, provision of financial incentives to improve adherence among adolescents, and potential ethical issues with this approach. Interview questionnaires and focus group discussion guides were designed with and conducted by youth leaders ages 18-25 who delivered peer support at CHUK. Finally, we iterated upon several ART adherence program prototypes with two peer mentors and 12 adolescents to ultimately co-design a youth-friendly intervention program deemed both acceptable and feasible in this community. The intervention ultimately consisted of two components: financial incentives and peer-led life skills training.

\section{Financial incentives for ART adherence}

Small financial incentives were used to promote ART adherence to achieve virological suppression. Other approaches to monitor ART adherence more directly (e.g. patient recall, directly observed therapy, or adherence monitoring devices such as Wisepill) were considered but deemed inappropriate or suboptimal for this context. While the World Health Organization establishes the threshold for having an undetectable viral load at 50 copies/milliliter (copies/mL), our program classified viral load suppression as less than 20 copies $/ \mathrm{mL}$, consistent with current Rwandan guidelines at the time of the study. Viral load was tested during clinic appointments at baseline and 12 weeks follow-up, and recorded in the OpenMRS medical records system. Clinic attendance was monitored through review of medical charts and updates from clinic staff.

The incentives structure is summarized in Table 1. The incentives program combined an immediate financial reward with a long-term savings opportunity. Two thousand Rwandan Francs (RWF), approximately \$2 USD, was deemed an appropriate incentive for clinic attendance. Of this total amount, 500 RWF ( \$0.50) were deposited into participants' TigoCash mobile money short-term account, where funds were immediately accessible, and 1500 RWF ( \$1.50) were deposited into a long-term savings account upon completing the program. 
Table 1

Incentive Structure for YBank Program Participation

\begin{tabular}{|ll|}
\hline Incentive Type & Incentive Amount (RWF) \\
\hline Clinic attendance & 2000* \\
\hline Short-term account & 500 \\
\hline Long-term account & 1500 \\
\hline Suppressed viral load & $4000 *$ \\
\hline Short-term account & 1000 \\
\hline Long-term account & 3000 \\
\hline *Maximum of two such incentives during the pilot study \\
\hline RWF: Rwandan Francs \\
\hline
\end{tabular}

Furthermore, if participants demonstrated a suppressed viral load at a clinic appointment, the research assistant deposited an additional 1000 RWF ( \$1) into their mobile money short-term account and 3000 RWF ( \$3) into their longterm account. If viral load was not suppressed, participants were encouraged to work with their physician to determine whether social and behavioral support was needed to improve adherence, or whether they may require evaluation for drug resistance.

At enrollment, adolescents were given a card for recording savings accrued to their short and long-term accounts. These cards were updated at each study visit when a disbursement was made.

[Table 1: Incentive Structure for YBank Program Participation]

\section{Life skills training}

In life skills training sessions, adolescents received guidance from peers on economic empowerment, financial literacy, healthy relationships, and ART adherence. The training materials were developed based on existing financial literacy training programs designed for Rwandan youth by non-profit organizations (GirlHub and Partners in Health), with consultation from experts in adolescent education. Three sessions were held over the course of 12 weeks and covered topics such as spending and saving habits, building a small business, and fostering both professional and personal relationships. These group sessions were held at the clinics and integrated with existing peer support sessions to minimize additional travel time for youth.

\section{Methods}

\section{Program population and sites}

The intervention was implemented at two sites in Rwanda, CHUK in urban Kigali and Kirehe District Hospital in rural Kirehe, both of which provide free HIV care for adolescents. Participant eligibility criteria for the study included HIVpositive adolescents between the ages of 12 and 19 who had been prescribed ART and were enrolled in care at one of the study sites for at least one year. The clinics compiled a list of all eligible adolescents in their care, and 50 adolescents were selected through randomization at CHUK. All 22 adolescents eligible for the study were enrolled at the Kirehe District Hospital site given the relatively small numbers of eligible participants. Those selected were contacted by a research assistant to participate in the study. They were then invited to a one-on-one information session followed by an enrollment session if they consented to participate. Informed consent was obtained from participants ages 18-19, and 
both parental/guardian consent and informed assent were obtained for participants ages 12-17. Participants were compensated 2000 RWF ( \$2) for travel to the clinics to attend program activities in keeping with local policies.

Institutional review board approval was obtained from the Rwandan National Ethics Committee, the Committee for the Protection of Human Subjects at Harvard T.H. Chan School of Public Health, and the Committee for the Protection of Human Subjects at the University of California, Berkeley School of Public Health.

\section{Data collection}

A baseline survey was administered by trained research assistants to all program participants and their caregivers. The survey included questions on demographics, access to financial services, and HIV status (Table 2). 
Table 2

Demographic, Mobile Banking, and HIV

Diagnostic Factors

\begin{tabular}{|c|c|c|}
\hline & $\mathbf{n}$ & $\%$ \\
\hline \multicolumn{3}{|l|}{ Age (years) } \\
\hline $12-13$ & 16 & 22 \\
\hline $14-15$ & 20 & 27 \\
\hline $16-17$ & 15 & 21 \\
\hline $18-19$ & 21 & 30 \\
\hline \multicolumn{3}{|l|}{ Sex } \\
\hline Male & 37 & 51 \\
\hline Female & 35 & 49 \\
\hline \multicolumn{3}{|l|}{ School/employment status } \\
\hline Primary school & 32 & 45 \\
\hline Secondary school (boarding) & 11 & 15 \\
\hline Secondary school (day) & 23 & 32 \\
\hline Employed & 3 & 4 \\
\hline Not in education or employment & 3 & 4 \\
\hline \multicolumn{3}{|l|}{ Family circumstances } \\
\hline Orphaned & 13 & 18 \\
\hline One parent alive & 25 & 35 \\
\hline Both parents alive & 34 & 47 \\
\hline \multicolumn{3}{|l|}{ Phone ownership } \\
\hline Yes & 42 & 58 \\
\hline No & 30 & 42 \\
\hline \multicolumn{3}{|l|}{ Banking activity } \\
\hline Currently have account & 5 & 7 \\
\hline Never had account & 66 & 92 \\
\hline Do not know & 1 & 1 \\
\hline \multicolumn{3}{|l|}{ Route of HIV transmission } \\
\hline Suspected perinatal & 63 & 88 \\
\hline Sexual & 2 & 3 \\
\hline Unknown & 7 & 9 \\
\hline Years since diagnosis & & \\
\hline
\end{tabular}




\begin{tabular}{|lll|}
\hline & $\mathbf{n}$ & $\%$ \\
\hline$<2$ years & 5 & 7 \\
\hline $2-4$ years & 25 & 35 \\
\hline $5-9$ years & 33 & 46 \\
\hline $10+$ years & 9 & 12 \\
\hline Viral load suppression at baseline & & \\
\hline Suppressed & 38 & 53 \\
\hline Not suppressed & 34 & 47 \\
\hline Clinic location & & \\
\hline Urban (CHUK) & 50 & 70 \\
\hline Rural (Kirehe) & 22 & 30 \\
\hline
\end{tabular}

Semi-structured interviews were conducted with 20 adolescents, 10 caregivers and two health workers randomly selected from each site during and directly following the intervention period. Broad themes explored in the interviews included experiences with receiving incentives and attending trainings, the impact of the intervention on caregivers and adolescents, and how the incentives were spent. Face-to-face interviews were conducted in Kinyarwanda or French by trained interviewers. The audio-recorded interviews lasted approximately 60 minutes and were translated into English for the study team. The recordings and transcriptions were uploaded to a password-protected cloud server.

Given that this was a pilot study assessing feasibility and acceptability of the intervention prior to a larger-scale study, the sample was not powered to statistically evaluate changes in virological suppression from pre to post-intervention.

\section{Data analysis}

Descriptive statistics were calculated to summarize baseline characteristics of our study sample using Stata software version 15.0. Qualitative data analysis using NVivo software version 10.0 involved initial open coding of interviews and focus groups to develop the codebook, whereby members of the study team read a subset of transcripts independently and carried out line-by-line coding. They then met and reviewed coding to discuss discrepancies, and refined the codebook through an iterative process until at least $90 \%$ interrater reliability was reached. At least two researchers carried out coding at any given time. This was followed by thematic analysis to identify patterns across interviews and create groupings of codes in order to answer our research questions regarding the acceptability and feasibility of the intervention [28]. Members of the study team reviewed translations of interview guides to ascertain consistency and validity. In addition, research assistants randomly selected recordings from each stakeholder group to ensure that transcriptions accurately reflected participants' statement in Kinyarwanda.

\section{Results}

\section{Descriptive statistics}

Seventy-two adolescents participated in the YBank intervention program from October 2016 to December 2016. During the 12-week study period, there was no loss to follow up. The vast majority (92\%) of participants were enrolled in either boarding or day school. Over half (53\%) had previously lost at least one parent. While $58 \%$ of participants owned a phone, less than $10 \%$ had a bank account through which they could receive mobile money. The majority (88\%) of HIV 
infections were thought to be passed perinatally, and $58 \%$ of participants had been formally diagnosed with HIV five or more years prior.

[Table 2: Demographic, Mobile Banking, and HIV Diagnostic Factors]

\section{Feasibility}

The study highlighted several operational challenges that could impact the feasibility of the intervention at scale. The program relied on enrolling adolescents into a mobile money platform to receive incentives. Fourteen adolescents reported challenges with registering their SIM cards, and three reported challenges using their PIN numbers to access TigoCash. Mobile phone use was relatively low among adolescents, especially those in rural areas; therefore, some participants borrowed phones. Adolescents under the age of 16 or without a national identification card were required to register their SIM card and mobile money accounts under the name of a caregiver, which posed an inconvenience to caregivers. When caregivers did not have time for registration, enrollment was delayed. These logistical challenges with mobile money usage are illustrated in following the quotes:

"My child forgot the PIN for the mobile money account and so could not access it. It was difficult to get back into it. I think she did not realize they need to remember it."

- Caregiver

"She uses the SIM card in her brother's cell phone. Mine was broken and sometimes it becomes difficult for her to use the SIM card when the brother has gone to boarding school. She is saving for a mobile phone but it is difficult."

- Caregiver

However, once successfully enrolled in the financial program, continued engagement and implementation of the program was feasible, with few reported operational challenges. Although it was anticipated that the intervention may have a negative effect on clinic workflow, no clinician interviewed expressed concerns about the impact of the study on their work.

In terms of program attendance, $75 \%$ (54/72) of adolescents attended all three of life skills training sessions, and $96 \%$ $(69 / 72)$ attended at least two out of three. Adolescents reported convenience of scheduling sessions on the weekends; however, exams caused some of those in boarding schools to miss sessions. We opted to add life-skills sessions to existing peer-support groups meetings to minimize multiple trips to the clinic. However, this led to the combined sessions exceeding the previously allotted two hours, based on feedback from study staff.

\section{Acceptability}

The program showed good acceptability among adolescents, caregivers, and health workers in semi-structured interviews. Adolescents expressed that they appreciated the combination of life skills training and rewards for medication adherence. Participants and caregivers expressed a desire to see other adolescents in HIV care benefit from this intervention as well. Four primary factors were identified as influencing acceptability of the intervention. These include psychosocial, financial, and health benefits, as well as interpersonal relationships.

Psychosocial benefits:

Adolescents described feeling encouraged, appreciated, and motivated upon receiving the financial incentives. They also expressed that the program gave them something to look forward to at each appointment. Some noted that it also helped counteract the negative emotional feelings normally associated with thinking about their HIV infection:

"No, there were no changes to my health or wellbeing since starting the program. I only gained new life skills and encouragement to keep taking the medicine properly. In addition, the program helped us to avoid isolation."

Page $10 / 17$ 


\section{- Participant, CHUK, 12, Male}

"The program helped me to overcome despair. There is a time when someone who is HIV-positive feels negative about him/herself. So, the program helped me to avoid stress and hopelessness."

\section{- Participant, CHUK, 18, Male}

Similarly, caregivers had positive opinions of the intervention, particularly around the changes and transformations in health behavior and mood of adolescents:

"My child can smile now, and she is self-confident compared to her mood before joining the program."

\section{- Caregiver}

"Actually, I have seen that he has changed, and I would say that he feels more positive about himself compared to some months before. He used to be shy, but now he is active and courageous. We can sit and talk about his life and encourage him to take the pills properly, while it was not the case in the previous days."

\section{- Caregiver}

Financial benefits:

Some adolescents shared that their ability to make a financial contribution to their own costs, such as school fees or transportation, alleviated economic tensions in the household. A number of adolescents also saved during the program and purchased equipment to start a business:

"I have saved 7500 Rwandan Francs. I have withdrawn the money and bought clothes with it. Now, I am saving again so that I can buy a sewing machine."

\section{- Participant, Kirehe, 15, Male}

"It could happen to me to spend a whole week without touching 500 Rwandan Francs, but you gave us some money when we came for training. Some of us were worried about our future, saying, "Other people are doing businesses and earning some money to spend on their daily needs. What will we do to get the chance also?" You finally came as a solution to our problems. You provided us with transport fees, and opened TigoCash accounts for us. We can save some money from the transport fee we receive from you. The money we receive from you will help us to start small businesses that will generate income for us."

\section{- Participant, Kirehe, 15, Male}

The savings component of the incentive was popular among caregivers who felt this encouraged saving and planning for the future:

"The program has a positive impact. Both the adolescents and us have appreciated the program. The adolescents like the money you have transferred to their TigoCash accounts. The adolescents say that they use a part of the money and save another part. We like this idea because the savings will help us sometime in the future."

\section{- Caregiver}

"There are many changes. Before joining the program, I couldn't afford school materials such as uniforms, shoes, and notebooks hardly; but today, as the child receives incentives and saves the money, we can afford them easily."

\section{- Caregiver}

Health benefits: 
Several adolescents, caregivers, and health workers discussed the perceived positive effect of the program on healthrelated behaviors or outcomes, including improvements in activity levels and overall sense of well-being:

"I used to be very thin and weak, and people could tell that I take medicines and do not eat. Now, I feel better and strong enough to do anything."

\section{- Participant, CHUK, 14, Female}

"I have noticed some changes. I met with peer mentors who encouraged me to take my pills more regularly, and on time. I have changed the way I had been doing; I am now taking the pills on time, and I feel better now."

\section{- Participant, CHUK, 14, Female}

"My child's health is improving, and she is now more active in the support group. The peer mentors share their experience with the young adolescents, and then later, they get restored and positive about their future. Caregivers do not set time to talk to their children about the problem they have, or some other caregivers are shy to tell their children why they take medicines every day, so the peer mentors do it well and counsel the young adolescents."

\section{- Caregiver}

Health workers also described the positive effect of the program on adolescents' adherence to medication and engagement in their own HIV care, including a renewed sense of agency in their treatment planning:

"I have noticed that the children have opened their minds compared to four months before. They are now active and respecting the medical appointments. Those who missed viral load testing before, they came during the pilot study. In brief, I would say that the program encouraged the children to adhere to their treatment and this has helped us as clinic staff to manage the children well, and they are now open to tell us the problems they face and why they may miss some medical appointments. It is easier now for us to find them when we need them."

\section{- Health provider}

Improvement in interpersonal relationships:

Caregivers described that, before the program, adolescents could not talk to them easily and did not follow their advice. However, the program improved communication, trust, and adolescents' resolve to save money:

"Before, I could not talk to her easily. She could not follow any of my pieces of advice. She was always mean to me. Today, when she arrives at home, she comes to me and shows me the money she has received and asks me to keep the SIM card for her. She now trusts in me. We have a common understanding about what she will spend the money on."

\section{- Caregiver}

\section{Discussion}

To our knowledge, YBank is the first program in sub-Saharan Africa to pilot the use of cash transfers and peer-led life skills training to improve HIV outcomes among adolescents. YBank is unique and novel in that we sought to integrate the needs of HIV-positive adolescents and their caregivers in designing an intervention that emphasizes economic independence along with physical and mental health promotion. This pilot study has key implications for program scaleup in a context where youth, especially those living with HIV, experience significant challenges in finding meaningful employment.

Interviews with adolescents and their caregivers revealed that the program was deemed acceptable and feasible overall. Specifically, adolescents reported that financial incentives enabled them to pay for transport to the clinic and afford their 
basic needs. In some instances, this reduced relational tensions in the home. These findings are consistent with that of Greene and colleagues, where a similar intervention on improving ART adherence among adults allowed patients to use incentives to address their fiscal needs [29]. The high levels of youth engagement in peer-led life skills trainings further demonstrates the acceptability of the program, as $75 \%$ of adolescents attended all of the life skills trainings and $96 \%$ attended two or more sessions.

Historically, adolescents have been excluded from the planning of cash transfer programs meant for them, often due to perceptions and notions that young people have poor judgment regarding finances and display risky spending behaviors [30]. This was an important consideration in our study, as we wanted to catalyze adolescents' agency in financial decision-making. Our findings indicated that the provision of financial incentives did not result in misuse of funds, and instead showed that the combination of training and cash incentives can reduce risky spending and increase savings.

Ultimately, the mobile money platform was accessible to all adolescents; however, registration of accounts and timely disbursement of funds was a significant challenge. Scaling of such an intervention will require integration of clinic attendance data and disbursement of financial incentives. Although there was low ownership of mobile phones and bank accounts among adolescents in our study, it is anticipated that technological access and literacy will only increase in Rwanda for future scale-up of the intervention. Additional effectiveness trials are recommended to understand through which pathways financial incentives best promote medication adherence among adolescents.

Several limitations of this study should be noted. First, interview participants were drawn from a convenience sample from only two clinics and, thus may not be representative of the wider population of HIV-infected adolescents. However, this is the common practice with qualitative studies, where the goal is to explore certain topics further in a targeted population, rather than obtain large, representative samples. This is a pilot study examining acceptability and feasibility of an intervention, and as such, is meant to inform the design and implementation of the scaled-up intervention, the internal and external validity of which can be studied using a randomized controlled trial in the future.

Second, patients and caregivers may have been more likely to participate in the program given the overall levels of poverty in the population, and more likely to offer positive feedback. We attempted to mitigate this issue by explaining that all the information provided would be anonymous and confidential, and that they were encouraged to give honest feedback.

Finally, given that Rwanda has a more robust healthcare infrastructure than many developing countries, and that the majority of HIV infections are transmitted perinatally, the condition may be less stigmatized and easier to manage

compared to other contexts. Thus, the findings from our study and program may not be generalizable to settings with weak healthcare infrastructure and higher rates of HIV infections transmitted sexually.

\section{Conclusions}

This is the first study to examine the feasibility and acceptability of a financial incentives and peer-led life skills training intervention to improve ART adherence among adolescents living with HIV in Rwanda. The implementation of such an intervention can have immense clinical significance, as medication adherence rates among adolescents in this region remain low despite access to ART treatment. In order to ensure treatment success, it is important to design strategies that address adolescents' unique socioeconomic challenges and involve them in the decision-making around their health care treatment and financial planning.

\section{Abbreviations}


Anti-retroviral therapy

CHUK

Centre Hospitalier Universitaire de Kigali

HIV/AIDS

Human Immunodeficiency Virus/Acquired Immunodeficiency Syndrome

$\mathrm{mL}$

Milliliter

RWF

Rwandan Francs

\section{Declarations}

\section{Competing interests:}

The authors declare that they have no competing interests.

\section{Funding:}

This study was supported by a grant from the Weiss Family Program Fund for Research in Development Economics.

\section{Acknowledgements}

We would like to thank the CRI Foundation, the Weiss Family Program Fund and the Harvard T.H. Chan School of Public Health, and the Royal College of Surgeons of England for their support in enabling us to undertake this study. We would also like to thank our research team, without whom this study would not have been possible: Alice Beardmore-Gray, Marie Aimee Umutoni Dusabe and Octave Irakarama (RAs - Kirehe); Juliette Mukasafari and Evelyn Isugi (RAs - CHUK); Delphine Niyotwagira and Nelson Turimana (PMs - Kirehe); Marie Jeanne Uwihirwe and Jean Claude de Golay Niyitanga (PMs - CHUK); Nour Sharara, Justin Healy, and Grace Wyshak (Harvard T. H. Chan School of Public Health).

\section{Ethics approval and consent to participate:}

This study was approved by the Rwandan National Ethics Committee, the Committee for the Protection of Human Subjects at Harvard T.H. Chan School of Public Health, and the Committee for the Protection of Human Subjects at the University of California, Berkeley School of Public Health. All study participants over the age of 18 provided written informed consent. All study participant ages 12-17 provided written informed assent as well as written parental consent.

\section{Consent for publication:}

Informed consent (and assent, where appropriate) was obtained from all individual participants included in the study.

\section{Availability of data and materials:}

The dataset generated and analyzed during the current study is not publicly available due to the sensitive nature of the subject matter but is available from the corresponding author on reasonable request. 


\section{Author contributions}

MO: Conception and design of project; acquisition, analysis, and interpretation of data; composition of manuscript SPN: Conception and design of project; acquisition, analysis, and interpretation of data; composition of manuscript MS: Conception and design of project; acquisition, analysis, and interpretation of data; composition of manuscript $\mathrm{RH}$ : Conception and design of the project; acquisition, analysis, and interpretation of data; composition of manuscript SM: Conception and design of the project; acquisition, analysis, and interpretation of data; composition of manuscript

$\mathrm{JL}$ : Analysis and interpretation of data; composition of manuscript

CK: Analysis and interpretation of data; composition of manuscript

TC: Design of project; acquisition, analysis, and interpretation of data

NP: Acquisition of data

BK: Acquisition of data

MO: Acquisition of data

MR: Conception and design of project

LIN: Acquisition, analysis, and interpretation of data

JLN: Conception and design of the project; acquisition, analysis, and interpretation of data

LH: Design of the study; acquisition of data

ER: Conception and design of the project

SN: Conception and design of the project

All authors read and approved the final manuscript.

\section{Authors' information}

Not applicable.

\section{References}

1. UNAIDS Data 2019. 2019. https://www.unaids.org/sites/default/files/media_asset/2019-UNAIDS-data_en.pdf.

2. Davies M-A, Pinto J. Targeting 90-90-90 - don't leave children and adolescents behind. J Int AIDS Soc. 2015;18:20745. doi:10.7448/IAS.18.7.20745.

3. Lowenthal ED, Bakeera-Kitaka S, Marukutira T, Chapman J, Goldrath K, Ferrand RA. Perinatally acquired HIV infection in adolescents from sub-Saharan Africa: a review of emerging challenges. Lancet Infect Dis. 2014;14:627-39. doi:https://doi.org/10.1016/S1473-3099(13)70363-3.

4. Ammon N, Mason S, Corkery JM. Factors impacting antiretroviral therapy adherence among human immunodeficiency virus-positive adolescents in Sub-Saharan Africa: a systematic review. Public Health. 2018;157:20-31. 
doi:https://doi.org/10.1016/j.puhe.2017.12.010.

5. Hudelson C, Cluver L. Factors associated with adherence to antiretroviral therapy among adolescents living with HIV/AIDS in low- and middle-income countries: a systematic review. AIDS Care. 2015;27:805-16.

doi:10.1080/09540121.2015.1011073.

6. Ryscavage PA, Anderson EJ, Sutton SH, Reddy S, Taiwo B. Clinical Outcomes of Adolescents and Young Adults in Adult HIV Care. JAIDS J Acquir Immune Defic Syndr. 2011;58.

https://journals.Iww.com/jaids/Fulltext/2011/10010/Clinical_Outcomes_of_Adolescents_and_Young_Adults.12.aspx.

7. Taddeo D, Egedy M, Frappier J-Y. Adherence to treatment in adolescents. Paediatr Child Health. 2008;13:19-24. doi:10.1093/pch/13.1.19.

8. Deteriorating Diabetes Control during Adolescence: Physiological or Psychosocial? . Journal of Pediatric Endocrinology and Metabolism . 2002;15:115. doi:10.1515/JPEM.2002.15.2.115.

9. Hamilton, J., and Daneman D. Deteriorating diabetes control during adolescence: physiological or psychosocial? J Pediatr Endocrinol Metab. 2002;15:115-26.

10. McClure SM, Laibson DI, Loewenstein G, Cohen JD. Separate Neural Systems Value Immediate and Delayed Monetary Rewards. Science (80- ). 2004;306:503 LP - 507. doi:10.1126/science.1100907.

11. Steinberg L. A social neuroscience perspective on adolescent risk-taking. Dev Rev. 2008;28:78-106. doi:https://doi.org/10.1016/j.dr.2007.08.002.

12. Huang Y, Hu P, Li X. Undervaluing delayed rewards explains adolescents' impulsivity in inter-temporal choice: an ERP study. Sci Rep. 2017;7:42631. doi:10.1038/srep42631.

13. Animasahun VJ, Sholeye 00, Oyewole BK. Transactional Sex Among Women Accessing Antiretroviral Care in Sagamu, Nigeria: A Descriptive Survey. Int Q Community Health Educ. 2018;39:225-31.

doi:10.1177/0272684X18821303.

14. Baral S, Beyrer C, Muessig K, Poteat T, Wirtz AL, Decker MR, et al. Burden of HIV among female sex workers in lowincome and middle-income countries: a systematic review and meta-analysis. Lancet Infect Dis. 2012;12:538-49.

doi:https://doi.org/10.1016/S1473-3099(12)70066-X.

15. Ssewamala FM, Han C-K, Neilands TB, Ismayilova L, Sperber E. Effect of Economic Assets on Sexual Risk-Taking Intentions Among Orphaned Adolescents in Uganda. Am J Public Health. 2010;100:483-8.

doi:10.2105/AJPH.2008.158840.

16. Ssewamala FM, Sensoy Bahar O, Tozan Y, Nabunya P, Mayo-Wilson LJ, Kiyingi J, et al. A combination intervention addressing sexual risk-taking behaviors among vulnerable women in Uganda: study protocol for a cluster randomized clinical trial. BMC Womens Health. 2019;19:111. doi:10.1186/s12905-019-0807-1.

17. Mutwa PR, Boer KR, Rusine J, Muganga N, Tuyishimire D, Schuurman R, et al. Long-term Effectiveness of Combination Antiretroviral Therapy and Prevalence of HIV Drug Resistance in HIV-1-infected Children and Adolescents in Rwanda. Pediatr Infect Dis J. 2014;33.

https://journals.Iww.com/pidj/Fulltext/2014/01000/Long_term_Effectiveness_of_Combination.15.aspx.

18. MacCarthy S, Saya U, Samba C, Birungi J, Okoboi S, Linnemayr S. "How am I going to live?": exploring barriers to ART adherence among adolescents and young adults living with HIV in Uganda. BMC Public Health. 2018;18:1158. doi:10.1186/s12889-018-6048-7.

19. Heise L, Lutz B, Ranganathan M, Watts C. Cash transfers for HIV prevention: considering their potential. J Int AIDS Soc. 2013;16:18615. doi:10.7448/IAS.16.1.18615.

20. Operario, D., Kuo, C., Sosa-Rubí, S. G., \& Gálarraga O. Conditional economic incentives for reducing HIV risk behaviors: integration of psychology and behavioral economics. Heal Psychol. 2010;100:483-8.

21. Pettifor AE, Rees H V, Kleinschmidt I, Steffenson AE, MacPhail C, Hlongwa-Madikizela L, et al. Young people's sexual health in South Africa: HIV prevalence and sexual behaviors from a nationally representative household survey. AIDS. 2005;19.

https://journals.Iww.com/aidsonline/Fulltext/2005/09230/Young_people_s_sexual_health_in_South_Africa_HIV.12.aspx. 
22. Ekwunife Ol, Anetoh MU, Kalu SO, Ele PU, Eleje GU. Conditional economic incentives and motivational interviewing to improve adolescents' retention in HIV care and adherence to antiretroviral therapy in Southeast Nigeria: study protocol for a cluster randomised trial. Trials. 2018;19:710. doi:10.1186/s13063-018-3095-4.

23. Mills EJ, Adhvaryu A, Jakiela P, Birungi J, Okoboi S, Chimulwa TNW, et al. Unconditional cash transfers for clinical and economic outcomes among HIV-affected Ugandan households. AIDS. 2018;32.

https://journals.Iww.com/aidsonline/Fulltext/2018/09100/Unconditional_cash_transfers_for_clinical_and.13.aspx. 24. Mahat G, Scoloveno MAVO-32. Effectiveness of Adolescent Peer Education Programs on Reducing HIV/STI Risk: An Integrated Review. Res Theory Nurs Pr. :168-2018. doi:10.1891/1541-6577.32.2.168.

25. Nabunya P, Ssewamala FM, Mukasa MN, Byansi W, Nattabi J. Peer mentorship program on HIV/AIDS knowledge, beliefs, and prevention attitudes among orphaned adolescents: an evidence based practice. Vulnerable Child Youth Stud. 2015;10:345-56. doi:10.1080/17450128.2015.1115157.

26. Mash R, Mash RJ. A quasi-experimental evaluation of an HIV prevention programme by peer education in the Anglican Church of the Western Cape, South Africa. BMJ Open. 2012;2:e000638. doi:10.1136/bmjopen-2011-000638. 27. Menna T, Ali A, Worku A. Effects of peer education intervention on HIV/AIDS related sexual behaviors of secondary school students in Addis Ababa, Ethiopia: a quasi-experimental study. Reprod Health. 2015;12:84. doi:10.1186/s12978015-0077-9.

28. RE B. Transforming Qualitative Information: Thematic Analysis and Code Development. Sage; 1998.

29. Greene E, Pack A, Stanton J, Shelus V, Tolley EE, Taylor J, et al. "It Makes You Feel Like Someone Cares" acceptability of a financial incentive intervention for HIV viral suppression in the HPTN 065 (TLC-Plus) study. PLoS One. 2017;12:e0170686. https://doi.org/10.1371/journal.pone.0170686.

30. Healy J, Hope R, Bhabha J, Eyal N. Paying for antiretroviral adherence: is it unethical when the patient is an adolescent? J Med Ethics. 2017;43:145 LP - 149. doi:10.1136/medethics-2015-103359. 